\title{
CRITÉRIO TÉCNICO PARA DETERMINAÇÃO DA IDADE ÓTIMA DE MUDAS DE EUCALIPTO PARA PLANTIO ${ }^{1}$
}

\author{
Reginaldo Gonçalves Mafia ${ }^{2}$, Acelino Couto Alfenas ${ }^{2}$, Leandro de Siqueira ${ }^{3}$, Eraclides Maria Ferreira², \\ Hélio Garcia Leite ${ }^{4}$ e José Renato P. Cavallazzi²
}

\begin{abstract}
RESUMO - Atualmente, a produção de mudas de Eucalyptus, em larga escala, é realizada pela miniestaquia, sob condições controladas. Todavia, ainda hoje não existe um critério técnico para determinar a idade ótima das mudas para plantio, o que pode refletir negativamente sobre a qualidade, sobretudo do sistema radicular, e, conseqüentemente, grandes perdas podem ocorrer no campo. Assim, o presente trabalho estabeleceu um critério técnico com base nas curvas de velocidade de crescimento e no intercepto entre as curvas de incremento corrente diário e incremento médio diário das variáveis altura, biomassa radicular e da parte aérea. Em virtude da restrição de volume explorável pelo sistema radicular, constataram-se reduções significativas na velocidade de crescimento das mudas que devem ser plantadas, preferencialmente, com 80 (clone A) a 100 (clone B) dias de idade, uma vez que mudas mais velhas sofrem redução na velocidade de crescimento e tendem a desenvolver problemas de malformação radicular. As implicações da baixa qualidade do sistema radicular residem na mortalidade de plantas, principalmente em períodos menos favoráveis, e na conseqüente influência na operação de replantio, bem como na realização dos testes clonais e na maior suscetibilidade das plantas aos estresses do ambiente.
\end{abstract}

Palavras-chave: Qualidade de mudas, eucalipto e sistema radicular.

\section{TECHNICAL CRITERION FOR THE DETERMINATION OF THE OPTIMUM AGE OF EUCALYPT CUTTINGS FOR PLANTING}

\begin{abstract}
Presently, mass vegetative propagation of Eucalyptus is carried out by mini-cuttings, under controlled conditions. Despite the advances of this technique, there is no criterion to determine the optimum age of the cuttings for planting, which can reflect negatively on the quality, mainly on the root system, and consequently losses may occur in the field. Hence, in the present work was established a technical criteria based on the growth speed curves and intercrossing of daily current increasing curves and daily average increasing for the variables height, root, stem and shoot biomass. Due to the restriction of exploitable volume by the root system, significant reductions in the growth speed of cuttings were found, and they must be planted preferentially from 80 (clone A) to 100 (Clone B) days of age, once older cuttings undergo a reduction in the growth speed and tend to develop root malformation problems. The implications of the low quality of the root system are the mortality of plants in the field, mainly in less favorable periods, and the consequent influence on the replanting operation, as well as on the clonal tests and a greater susceptibility of plants to environment stresses.
\end{abstract}

Keywords: cuttings quality, eucalypt and root system.

\footnotetext{
${ }^{1}$ Recebido em 15.09.2004 e aceito para publicação em 10.08.2005.

${ }^{2}$ Departamento de Fitopatologia da UFV, 36570-000 Viçosa - MG.

${ }^{3}$ Cia. Suzano Bahia Sul S.A., 18200-000 Itapetininga - SP.

${ }^{4}$ Departamento de Engenharia Florestal da UFV, 36570-000 Viçosa - MG.
} 


\section{INTRODUÇÃO}

O Brasil possui, atualmente, a segunda maior área reflorestada com espécies de Eucalyptus do mundo, perfazendo um total aproximado de três milhões de ha, cuja madeira produzida é destinada, principalmente, à produção de polpa celulósica e carvão vegetal (CAMPINHOS JR., 1999; ELDRIDGE et al., 1994).

A produção de mudas de eucalipto é realizada, principalmente, pela propagação vegetativa. A clonagem do eucalipto iniciou-se em 1975 na República Popular do Congo (DELWAULLE et al., 1983) e foi introduzida no Brasil ainda na década de 1970 (CAMPINHOS e IKEMORI, 1983), o que proporcionou, dentre outras vantagens, a transferência de características genéticas pouco herdáveis, como o incremento em volume e o rendimento em celulose, possibilitando grandes avanços nos programas de melhoramento genético (PENCHEL et al., 1995). Desde sua introdução, a propagação clonal de Eucalyptus sofreu grandes avanços. Inicialmente, as mudas eram produzidas por meio do enraizamento de estacas, atualmente denominadas macroestacas, obtidas a partir de brotações de cepas estabelecidas em áreas de jardim clonal ou de bancos clonais após o corte raso (CAMPINHOS e IKEMORI, 1983; CAMPINHOS, 1987). Entretanto, a estaquia convencional, atualmente denominada macroestaquia, apresenta como um de seus obstáculos o baixo porcentual de enraizamento de alguns clones e as dificuldades inerentes aos tratos culturais, como irrigação, fertilização e controle de fitopatógenos, principalmente em períodos chuvosos (ALFENAS et al., 1997). Outro inconveniente é a possibilidade de ocorrência da redução gradual do potencial de enraizamento, com o envelhecimento ontogênico das matrizes (ASSIS, 1997). Com o objetivo de minimizar esses problemas, na década de 1990 foram desenvolvidas as técnicas de micro e miniestaquia (ASSIS et al., 1992; ASSIS, 1997; ASSIS, 2001), possibilitando a clonagem comercial de genótipos de difícil enraizamento. Atualmente, a miniestaquia tem sido a técnica mais empregada para produção de mudas em escala comercial, enquanto a microestaquia tem sido utilizada apenas para o rejuvenescimento de clones recalcitrantes ao enraizamento, quando se empregam técnicas de estaquia convencional e miniestaquia (TITON et al., 2002).

Ainda hoje, mesmo diante de toda a evolução ocorrida no processo de clonagem, desde o advento da técnica de macroestaquia não existe um critério técnico para determinar a idade ótima das mudas para plantio, o que tem refletido significativamente sobre a qualidade, sobretudo do sistema radicular. A malformação do sistema radicular impede a absorção de água e nutrientes em quantidades suficientes para atender às necessidades da planta, resultando em um quadro sintomatológico típico de deficiência hídrica e, ou, nutricional, em consequiência do desequilíbrio entre raiz e parte aérea. Esse problema está geralmente associado à deformação do sistema radicular de mudas na fase de viveiro ou no ato do plantio, à falta de adaptação da espécie para a região e aos plantios em solos compactados e, ou, sujeitos ao encharcamento (ALFENAS et al., 2004).

O tipo e o número de estrias de direcionamento nos tubetes, bem como a idade das mudas, afetam a arquitetura do sistema radicular e, conseqüentemente, o sucesso do plantio definitivo no campo. Uma das causas mais comuns da malformação do sistema radicular das mudas no viveiro é o uso de recipientes inadequados que promovem a distribuição anormal de raízes laterais e superficiais. No campo, as plantas afetadas exibem menor crescimento e, geralmente, em épocas de estiagem são levadas à morte em consequiência de enovelamentos, dobras e estrangulamentos das raízes. Os danos variam normalmente com a espécie, com o genótipo, com a idade da planta e com a disponibilidade de água e nutrientes no solo. Problemas de má-qualidade de mudas resultam em desuniformidade do talhão quanto ao crescimento das plantas. Além disso, plantas com sistema radicular superficial tornam-se mais vulneráveis aos efeitos deletérios de matocompetição e oscilação de temperatura e demandam maior disponibilidade de água e nutrientes no solo logo nos meses subseqüentes ao plantio. Ademais, plantas com o sistema radicular superficial tornam-se mais propícias à queda pelo vento. Em geral, esses efeitos se agravam a partir do segundo ano de plantio (ALFENAS et al., 2004).

O plantio de mudas velhas tem sido um dos maiores problemas para o estabelecimento e o arranque inicial dos plantios no campo. Isso porque, a partir de certa idade no viveiro, as mudas reduzem o seu crescimento vegetativo e tendem a enovelar o sistema radicular em virtude da restrição de volume explorável de substrato imposto pelo recipiente de produção de mudas. Dessa forma, o presente trabalho objetivou estabelecer um critério técnico para determinar a idade ótima de expedição de mudas para o campo, considerando-se a propagação por miniestaquia e empregando recipientes do tipo tubetes de $50 \mathrm{cc}$ de capacidade. 


\section{MATERIAL E MÉTODOS}

Miniestacas com 5,0 cm de comprimento, contendo dois pares de folhas seccionadas ao meio, foram coletadas de minijardim clonal de dois clones de eucalipto (híbrido de Eucalyptus grandis Hill ex Maiden x E. urophylla Blake) e estaqueadas em substrato de enraizamento composto de uma mistura de $50 \%$ de vermiculita tipo fina, 30\% de substrato orgânico (Mecplant florestal tipo 3) e $20 \%$ de palha de arroz carbonizada, enriquecido com $3 \mathrm{~kg} / \mathrm{m}^{3}$ de osmocote (10-5-20) e $6 \mathrm{~kg} / \mathrm{m}^{3} \mathrm{de}$ superfosfato simples. Após essa etapa, as miniestacas foram transferidas para casa de enraizamento dotada de nebulização intermitente de água, as quais foram mantidas por 30 dias. A partir de 30 dias, realizaramse avaliações periódicas em intervalos de cinco dias até que as mudas completassem 50 dias e, a partir daí, as avaliações foram realizadas em intervalos de 10 dias até a idade de 120 dias. Nas avaliações, quantificouse a altura da parte aérea, bem como a biomassa seca do sistema radicular e da parte aérea. Para se proceder às avaliações dessas duas últimas variáveis, cada muda teve seu sistema radicular separado da parte aérea e, após remover os resíduos de substrato, procedeu-se à secagem em estufa com ventilação forçada a $90^{\circ} \mathrm{C}$ por $24 \mathrm{~h}$. Em seguida, determinou-se a biomassa seca de cada parte da muda em balança de precisão.

O experimento foi montado, para cada clone, em delineamento inteiramente casualizado, composto de 10 repetições para cada tempo de avaliação, sendo

Quadro 1 - Parâmetros e coeficiente de correlação do modelo logístico empregado para explicar a relação entre altura média, biomassa da parte aérea e do sistema radicular de mudas de dois clones de eucalipto em função da idade

Table 1 - Parameters and coefficient of correlation of the logistic model employed to explain the relation between average height, shoot and stem biomass and the seedling root system of two clones of eucalypt in function of the age

\begin{tabular}{lccccc}
\hline Variável & Clone & \multicolumn{3}{c}{ Parâmetros do Modelo } & r \\
\cline { 3 - 5 } & & \multicolumn{3}{c}{$\mathrm{Y}=\alpha\left(1+\beta \mathrm{e}^{-\gamma \mathrm{T}}\right)^{-1}+\varepsilon$} & \\
\hline Altura & $\mathrm{A}$ & 30,7721 & 15,0804 & 0,0459 & 99,2 \\
& $\mathrm{~B}$ & 47,7521 & 13,8666 & 0,0327 & 99,0 \\
Biomassa da & $\mathrm{A}$ & 1205,4167 & 365,2770 & 0,0962 & 99,3 \\
parte aérea & $\mathrm{B}$ & 1446,5262 & 84,7867 & 0,0601 & 99,0 \\
Biomassa & $\mathrm{A}$ & 559,8727 & 449,2424 & 0,1141 & 99,7 \\
radicular & $\mathrm{B}$ & 566,7576 & 209,3402 & 0,1072 & 98,7 \\
\hline
\end{tabular}

cada muda representativa de uma repetição. Em cada variável, ajustou-se o modelo logístico (Quadro 1) definido por $\mathrm{Y}=\alpha\left(1+\beta \mathrm{e}^{-\gamma \mathrm{T}}\right)^{-1}+\varepsilon$, em que $\mathrm{Y}=$ variável avaliada e $\mathrm{T}=$ número de dias. A partir dos modelos de regressão, calcularam-se o incremento corrente diário, o incremento médio diário e a velocidade de crescimento, a fim de determinar a idade ótima de expedição, empregando-se a função $\mathrm{d} Y / \mathrm{d} T=\gamma x \mathrm{Y}(\alpha-\mathrm{Y}) / \alpha$. O procedimento estatístico, proposto por Leite e Oliveira (2002), foi utilizado para o teste de identidade de modelos, no nível de $5 \%$ de probabilidade.

\section{RESULTADOS}

Nos dois clones testados, observaram-se comportamentos similares quanto à dinâmica de crescimento, considerando altura (Figura 1) e biomassa da parte aérea (Figura 2) e do sistema radicular (Figura 3). Além disso, ficou evidenciada a ocorrência de estabilização no desenvolvimento do sistema radicular, imposto principalmente pela restrição de volume explorável $\left(50 \mathrm{~cm}^{3}\right)$ de substrato. Como reflexo dessa estabilização, verificou-se o mesmo comportamento na altura do clone $\mathrm{A}$.

Considerando como critério técnico para determinar a idade ótima das mudas para plantio o intercepto entre a curva de incremento corrente diário e a curva de incremento médio diário para cada variável, observouse no clone A que a idade de 82 dias foi definida como a mais indicada para altura média das mudas e para acúmulo de biomassa da parte aérea. Em relação à biomassa radicular, ficou definido que, a partir de 72 dias, ocorreu uma estabilização no desenvolvimento, evidenciado pelo intercepto entre o incremento médio diário e o incremento corrente diário. Para o clone B, a idade ótima de expedição, considerando esse critério, foi de 110 dias para altura média, 102 dias para biomassa da parte aérea e de 68 dias para biomassa radicular (Figura 4).

A partir da velocidade de crescimento, ficou evidenciado que, para altura média, o máximo ocorreu em 60 e 61 dias nos clones A e B (Figura 5), enquanto na biomassa da parte aérea o máximo aconteceu entre 63 e 70 dias (Figura 6), respectivamente. Considerando a velocidade de crescimento do sistema radicular, notouse que o máximo dessa variável ocorreu entre 51 e 55 dias nos clones B e A, respectivamente (Figura 7).

R. Árvore, Viçosa-MG, v.29, n.6, p.947-953, 2005 


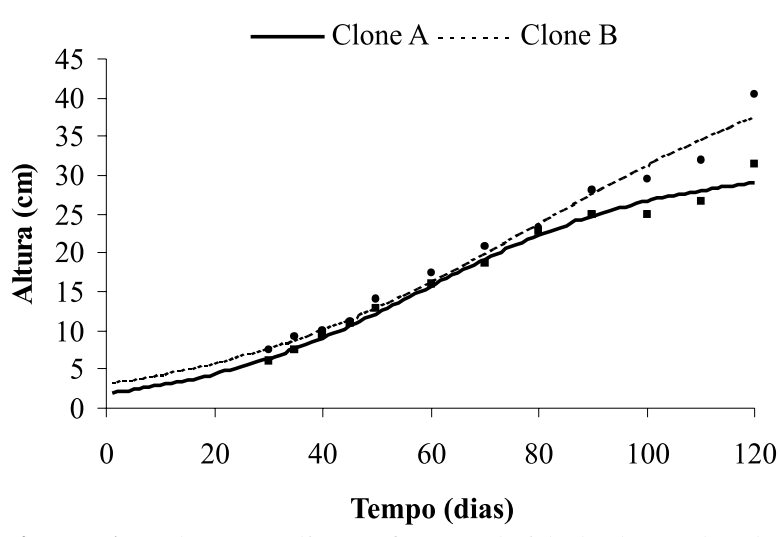

Figura 1 - Altura média em função da idade de mudas de dois clones de eucalipto.

Figure 1-Mean height according to the age of cuttings for two clones of eucalypt.

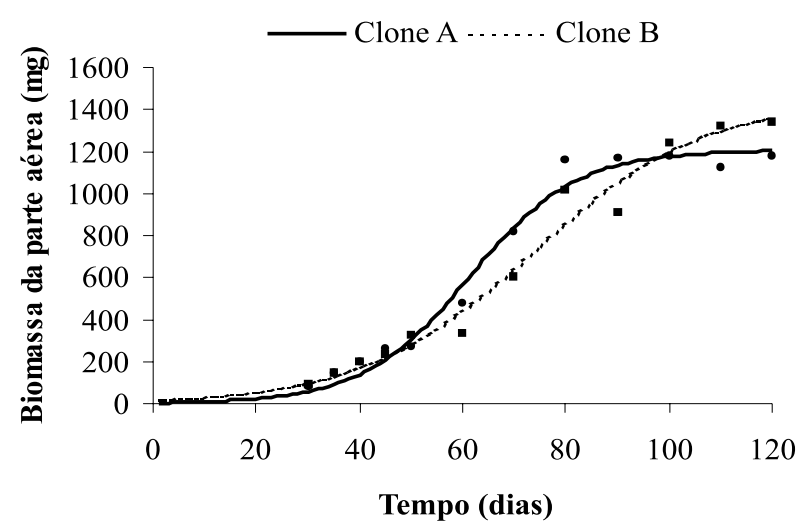

Figura 2 - Biomassa média da parte aérea em função da idade de mudas de dois clones de eucalipto.

Figure 2-Mean stem and shoot biomass according to the age of cuttings for two clones of eucalypt.

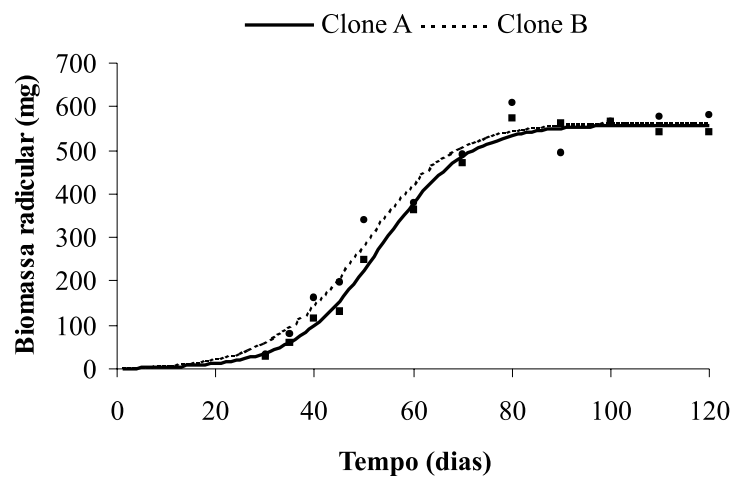

Figura 3 - Biomassa média radicular em função da idade de mudas de dois clones de eucalipto.

Figure 3-Mean root biomass according to the age of cuttings for two clones of eucalypt.

\section{DISCUSSÃO}

A qualidade das mudas é afetada, dentre outros fatores, pelo tempo de permanência no viveiro. Isso porque, ao ultrapassarem o período ideal de rotação no viveiro, as mudas tendem a apresentar enovelamento do sistema radicular, imposto pela restrição de espaço explorável de substrato, aliado a um baixo vigor vegetativo, redução de área foliar, maior predisposição a determinadas doenças etc. (ALFENAS et al., 2004). Ainda hoje não existe um método que permite determinar a idade ótima de expedição das mudas para plantio, sendo normalmente utilizado valores empíricos. Além do maior custo relativo à manutenção das mudas, existem várias implicações com a redução da qualidade do sistema radicular.

A primeira implicação direta da má-qualidade do sistema radicular refere-se à mortalidade a campo, principalmente em épocas menos favoráveis ao desenvolvimento das plantas. Como se sabe, o replantio é oneroso e a decisão de realizar ou não esta operação depende de vários fatores técnicos e econômicos. Dentre os fatores técnicos, merecem destaque o desenvolvimento e a idade do povoamento em que se pretende realizar o replantio. Isso porque, dependendo do tamanho das plantas no campo, o replantio promoverá uma competição desproporcional pelos fatores do ambiente entre as plantas remanescentes e as novas mudas, ocasionando índice elevado de mortalidade e desuniformidade do plantio, com o surgimento de plantas suprimidas. Plantas suprimidas são indesejáveis não somente pelo seu menor desenvolvimento, mas também por serem mais suscetíveis a pragas e doenças e por atuarem como fonte de inóculo em epidemias. Além disso, são mais vulneráveis às condições de estresse do ambiente, como secas, ventos etc. (ALFENAS et al., 2004).

Com relação aos fatores econômicos, o índice de mortalidade no campo exerce grande influência na decisão de realizar ou não o replantio. Como exemplo disso, em uma empresa situada no Estado de São Paulo o replantio é realizado sempre quando esse valor é superior a $3 \%$ e a idade do povoamento não ultrapassou os 30 dias. Assim, a mortalidade de plantas ocasionadas pela má-qualidade do sistema radicular passa a exercer grande influência no custo de implantação das florestas, podendo causar grandes perdas no campo. Dependendo da intensidade e da gravidade das más conformações do sistema radicular, as mortes poderão ocorrer em grande número e se manifestar no longo prazo, não sendo possível realizar a correção pelo replantio. 

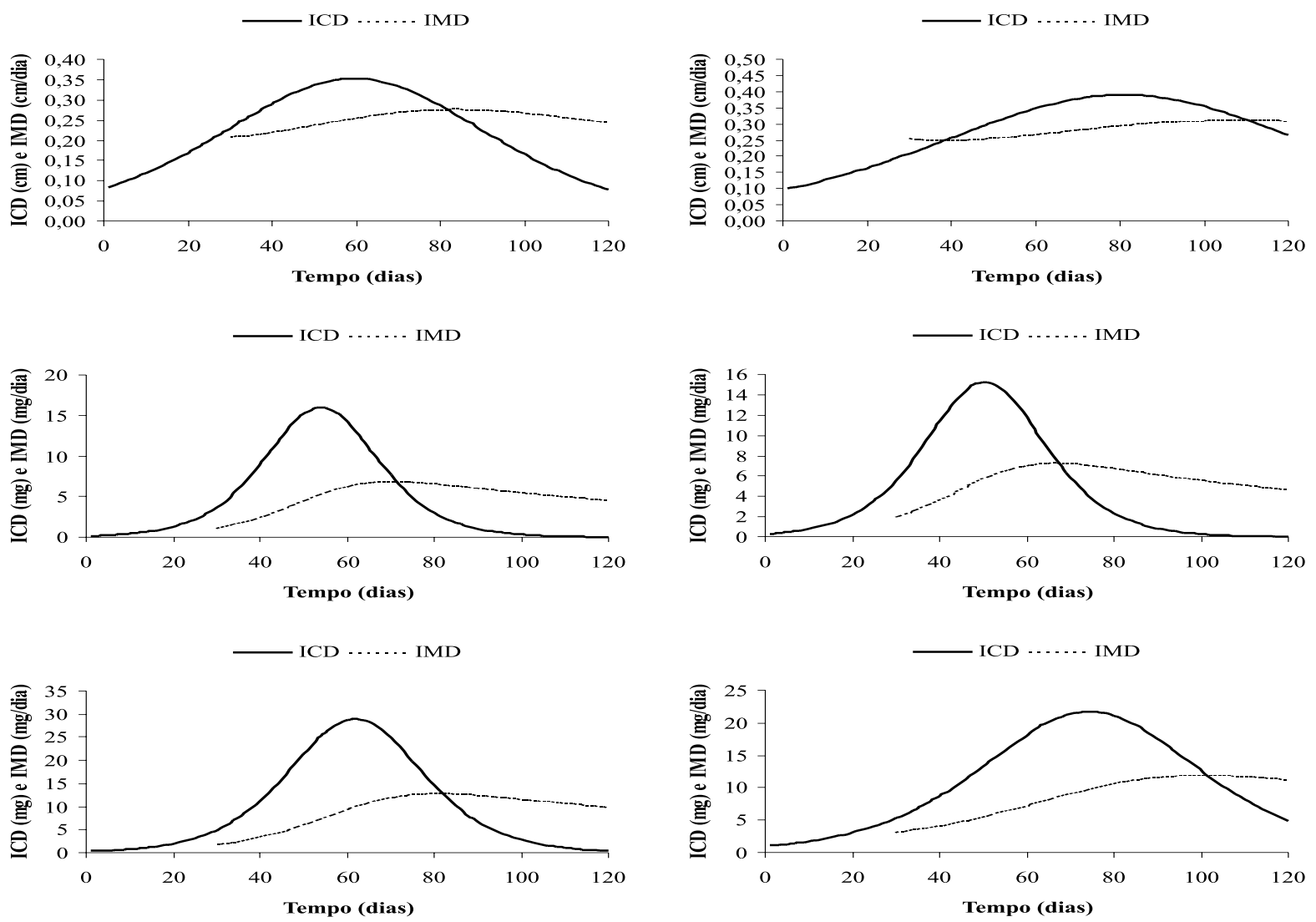

Figura 4 - Taxas de crescimento e incremento corrente diário (ICD) e incremento médio diário (IMD) para altura (parte superior) e biomassa da parte aérea (centro) e do sistema radicular (parte inferior), em função da idade de dois clones de eucalipto (clone $\mathrm{A}$, à esquerda e clone $\mathrm{B}$, à direita).

Figure 4-Daily current increment (DCI) and average daily increment indexes (ADI) for height (upper part) and stem and shoot (middle part) and root (lower part) biomass according to the age of two clones of eucalypt (clone $A$, left and clone B, right).

Clone A …... Clone B

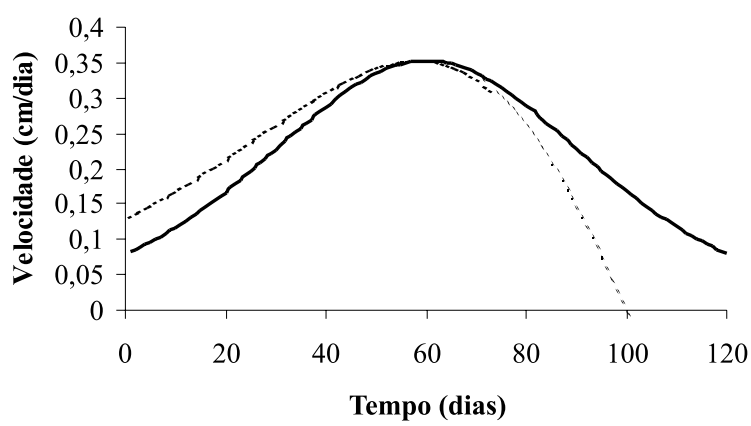

Figura 5 - Curva de crescimento considerando a altura média em função da idade de mudas de dois clones de eucalipto.

Figure 5 - Growth curve considering mean height according to the age of cuttings for two clones of eucalypt.

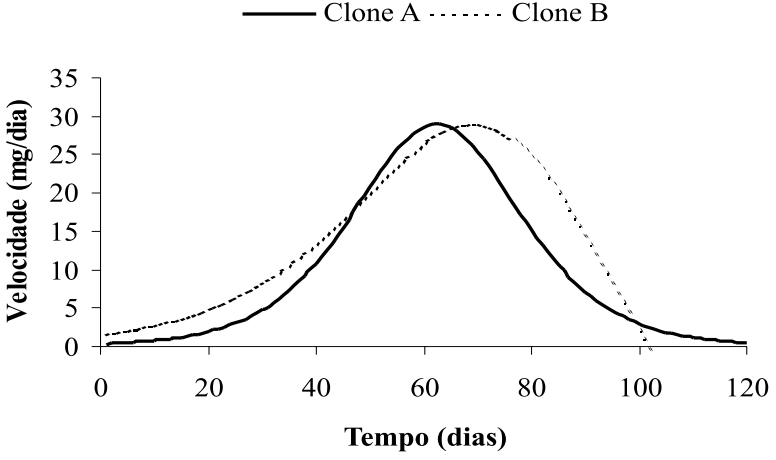

Figura 6 - Curva de crescimento considerando o acúmulo de biomassa da parte aérea em função da idade de dois clones de eucalipto.

Figure 6-Growth curve considering biomass accumulation of shoot and stem according to the age of cuttings for two clones of eucalypt.

R. Árvore, Viçosa-MG, v.29, n.6, p.947-953, 2005 


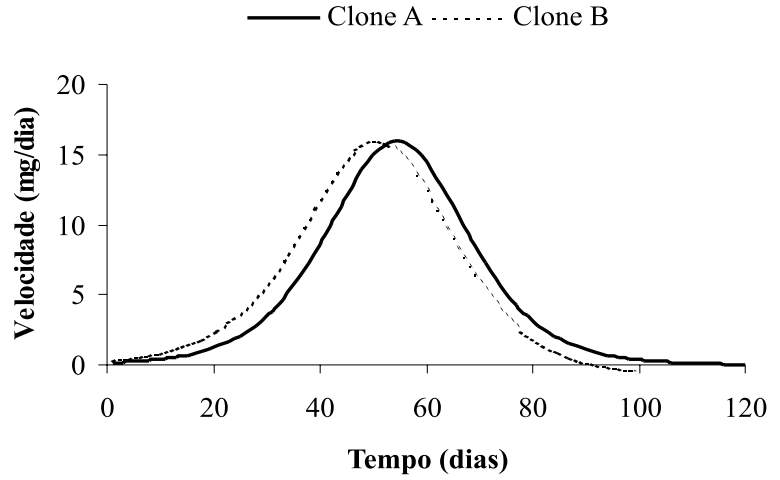

Figura 7 - Curva de crescimento considerando o acúmulo de biomassa radicular em função da idade de mudas de dois clones de eucalipto.

Figure 7 - Growth curve considering biomass accumulation of root according to the age of cuttings for two clones of eucalypt.

Outro ponto a ser considerado quanto à qualidade das mudas reside na realização dos testes clonais. É possível que nesses testes determinados clones possam não expressar todo o seu potencial genético e ser descartados, indevidamente, em razão da má-qualidade do sistema radicular, pois, nesses casos, o enovelamento de raízes poderá prejudicar o desenvolvimento da planta sem causar-lhe a morte, passando, dessa forma, desapercebido pelos melhoristas, principalmente na realização da seleção precoce.

Em um estudo desenvolvido por Mello et al. (1997), no qual se caracterizou o sistema radicular de povoamentos de eucalipto propagados por sementes e estacas, foi observado que a distribuição e a variação estacional da quantidade de raízes no perfil do solo apresentaram-se como características estreitamente relacionadas com a capacidade de adaptação de genótipos aos estresses hídrico e térmico do solo, afetando diretamente seus potenciais produtivos. Diante disso, é sempre recomendável considerar a qualidade das mudas nos testes clonais, principalmente em termos do sistema radicular.

Outra implicação direta da baixa qualidade do sistema radicular é o desequilíbrio elevado entre raiz e parte aérea. Em um estudo desenvolvido por Donoso et al. (2001) sobre o desenvolvimento e arquitetura do sistema radicular de plantios de E. globulus Labill, provenientes de estacas e sementes, ficou comprovado que a relação entre biomassa de raízes (BR) e biomassa aérea (BA) é extremamente inferior no plantio clonal, em comparação com o proveniente de sementes. Dessa forma, a menor relação BR/BA no plantio clonal, aliada à baixa qualidade da arquitetura do sistema radicular, poderá comprometer a estabilidade das árvores, tornando-as mais suscetíveis ao tombamento pelo vento.

\section{CONCLUSÕES}

Em razão da limitação de volume de substrato explorável e diante das variáveis analisadas, ficou evidenciado que o acúmulo de biomassa radicular ao longo do tempo é uma boa variável para dirimir dúvidas sobre o tempo ideal para determinar a idade ótima de mudas para plantio, considerando-se a manutenção do "status" vegetativo e da qualidade do sistema radicular.

Considerando a limitação de volume do substrato explorável e pelo critério técnico utilizado, concluise que, diante das variáveis analisadas, 80 (clone A) a 100 (clone B) dias são suficientes para expedição de mudas de boa qualidade em relação ao sistema radicular. Além disso, a manutenção de mudas por maiores períodos no viveiro tende a reduzir a velocidade de crescimento e induzir malformações radiculares.

Os critérios técnicos de intercepto entre as curvas de incremento corrente diário e incremento médio diário e do ponto de máximo da curva de velocidade de crescimento podem ser úteis para a tomada de decisão, com vistas à avaliação do padrão de qualidade de mudas.

\section{REFERÊNCIAS BIBLIOGRÁFICAS}

ALFENAS, A.C.; SILVEIRA, S.F.; SANFUENTES, E.A. Current status and control strategies of disease associated to clonal propagation of Eucalyptus in Brazil. In: IUFRO CONFERENCE ON SILVICULTURE AND IMPROVEMENT OF EUCALYPTUS, 1997, Colombo. Proceedings... Colombo: EMBRAPA/CNPF, 1997. v.4. p.106-111.

AlfEnAS, A.C. et al. Clonagem e doenças do eucalipto. Viçosa, MG: Universidade Federal de Viçosa, 2004. 442p.

ASSIS, T.F. Evolution of technology for cloning Eucalyptus in large scale. In: IUFRO INTERNATIONAL SYMPOSIUM, 2001, Valdivia. Proceedings... Chile: EMBRAPA. Centro Nacional de Pesquisas Florestais, 2001. v. 4. 
ASSIS, T.F. Propagação vegetativa de Eucalyptus por microestaquia. In: IUFRO CONFERENCE ON SILVIVULTURE AND IMPROVEMENT OF EUCALYPTS, 1997, Salvador. Proceedings... Colombo: EMBRAPA/CNPF, 1997. v.1. p.300-304.

ASSIS, T.F.; ROSA, O.P.; GONÇALVES, S.I. Propagação clonal de Eucalyptus por microestaquia. In: CONGRESSO FLORESTAL ESTADUAL, 7., 1992, Nova Prata. Anais... Santa Maria: Universidade Federal de Santa Maria, 1992. p.824.

CAMPINHOS JR., E. Sustainable plantations of high-yield Eucalyptus trees for production of fiber: the Aracruz case. New Forests, v.17, p.129-143, 1999.

CAMPINHOS, E. Propagacion vegetativa de Eucalyptus spp. por enraizametno de estacas. In: SIMPOSIO SOBRE SILVICULTURAY MEJORAMIENTO GENETICO DE ESPECIES FORESTALES, 1987, Buenos Aires. Anais... Buenos Aires: CIEF, 1987. v.1.p.208-214.

CAMPINHOS, E.; IKEMORI, Y.K. Introdução de novas técnicas na produção de mudas de essências florestais. Silvivultura, v.8, n.28, p.226-228, 1983.

DELWAULLE, J.C.; LAPLACE, Y.; QUILLET, G Production massive de boutures d' Eucalyptus en République Populaire du Congo. Silvivultura, v.8, n.32, p.779-781, 1983.
DONOSO, S.; RUIZ, F.; HERRERA, M.A.

Evaluación del desarrollo y arquitectura de raíces de plantaciones de Eucalyptus globulus provenientes de semilla y estaca. In: IUFRO INTERNATIONAL SYMPOSIUM, 2001, Valdivia. Proceedings... Chile: EMBRAPA. Centro Nacional de Pesquisas Florestais, 2001. v.4.

ELDRIDGE, K.G. et al. Eucalypt domestication and breeding. Oxford: Oxford Science Publications, Clarendon Press, 1994. 288 p.

LEITE, H.G.; OLIVEIRA, F.H.T. Statistical method to test the identity of analytical methods. Communications in Soil Science Plant Analysis, n.6, 7.p. 22, 2002.

MELLO, S.L. et al. Características do sistema radicular em povoamentos de eucaliptos propagados por sementes e estacas. In: IUFRO CONFERENCE ON SILVIVULTUREAND IMPROVEMENT OF EUCALYPTS, 1997, Salvador Proceedings... Colombo: EMBRAPA/CNPF, 1997. v.3, p.54-62.

PENCHEL, R.M. et al. Otimização de parâmetros fisiológicos da propagação vegetativa por estaquia de matrizes elite de eucaliptos. In: CONGRESSO BRASILEIRO DE FISIOLOGIA VEGETAL, 5., 1995, Lavras. Resumos... Lavras: Sociedade Brasileira de Fisiologia Vegetal, 1995. 83p.

TITON, M.; XAVIER, A.; OTONI, W.C. Dinâmica do enraizamento de microestacas e miniestacas de clones de Eucalyptus grandis. Revista Árvore, v.26, n.6, p.665-673, 2002. 